\title{
Mucopolysaccharidosis Type I in Children, a Forgotten Diagnosis Responsible for Undiagnosed Musculoskeletal Complaints: Report of Two Cases
}

\author{
Soheila Hoseinzadeh Moghadam¹, Masood Ghahvechi ${ }^{1,2}$, Fatemeh Mozafari ${ }^{1}$, Fatemeh Sayarifard ${ }^{3,4}$, \\ Mahdieh-Sadat Mousavi ${ }^{1,3}$, Reza Rostami ${ }^{5}$, Vahid Ziaee ${ }^{1,3,6, *}$
}

\begin{abstract}
Mucopolysaccharidoses (MPS) are a subgroup of lysosomal storage disorders. The underlying mechanism of MPS disorders are deficiency in specific enzymes which leads to accumulation of partially degraded glycosaminoglycans (GAGs) in various tissues. A wide variety of manifestations are reported but musculoskeletal complaints are common among them. In milder forms of MPS, musculoskeletal complaints are presenting symptoms. Delays in diagnosis due to unspecific and mild symptoms is common. Misdiagnosis of MPS as juvenile idiopathic arthritis and other inflammatory arthritis disorders is frequent. Early diagnosis and treatment prevents irreversible cellular damages and is a key factor in efficacy of enzyme replacement therapy. In this study we described two MPS patients with musculoskeletal complaints who were not diagnosed for a period of time. Although musculoskeletal manifestation are common in a variety of clinical conditions, their presence at low ages or co-occurrence of other manifestations (such as cardiac, respiratory, neurologic, etc.) in multiple systems should prompt evaluation of patients for MPS and other metabolic disorders. The rheumatologists' awareness on MPS should be promoted to achieve timely diagnosis and subsequent early treatment.
\end{abstract}

\section{KEYWORDS}

mucopolysaccharidoses; glycosaminoglycan; hand drop; juvenile idiopathic arthritis; pediatric rheumatology

AUTHOR AFFILIATIONS

${ }^{1}$ Children's Medical Center, Pediatrics Center of Excellence, Tehran, Iran

2 Department of Physical Medicine, Tehran University of Medical Sciences, Tehran, Iran

${ }^{3}$ Department of Pediatrics, Tehran University of Medical Sciences, Tehran, Iran

${ }^{4}$ Growth and Development Research Center, Tehran University of Medical Sciences, Tehran, Iran

${ }^{5}$ Department of Psychology and Education, School of Psychology and Education Science, University of Tehran, Tehran, Iran

${ }^{6}$ Pediatric Rheumatology Research Group, Rheumatology Research Center, Tehran University of Medical Sciences, Tehran, Iran

* Corresponding author: Division of Pediatric Rheumatology, Children's Medical Center, No. 62 Dr. Gharib St., Keshavarz Blvd, Tehran 14194, IR Iran; e-mail: ziaee@tums.ac.ir

Received: 24 May 2019

Accepted: 4 September 2019

Published online: 10 February 2020

Acta Medica (Hradec Králové) 2019; 62(4): 161-165

https://doi.org/10.14712/18059694.2020.6

(c) 2019 The Authors. This is an open-access article distributed under the terms of the Creative Commons Attribution License (http://creativecommons.org/licenses/by/4.0), which permits unrestricted use, distribution, and reproduction in any medium, provided the original author and source are credited. 


\section{INTRODUCTION}

Mucopolysaccharidoses (MPS) are a subgroup of lysosomal storage diseases which are caused by progressive deposition of glycosaminoglycans (GAGs) in tissues. This deposition is due to enzymatic defects which impair degradation of GAGs (1). Seven types of MPS are described with specific enzymatic deficiencies. In addition to the type of deficient enzyme, the amount of deficiency is correlated with severity of the disease. GAG accumulation in cells occurs in various organ systems which leads to markedly reduced lifespan, multiple organ failures and cognitive function deterioration (2). Musculoskeletal complaints are present in nearly all types of MPS and represent the most common presenting symptom in these patients (3). Skeletal dysplasia, decreased joint mobility, short stature and Carpal tunnel syndrome are among the most prevalent musculoskeletal symptoms in MPS (1). Due to extensive involvement of different body organs, variety of clinical manifestations and rarity of MPS, misdiagnosis and delay to diagnosis are frequent. Usually patients with musculoskeletal complaints are referred to rheumatologists and they are frequently diagnosed with more prevalent conditions such as Juvenile idiopathic arthritis (4). Some efficacious treatments are developed for specific types of MPS (including MPS type I which is the most common form of MPS). Enzyme replacement therapy has shown promising outcomes in patients suffering from MPS type I. The most important factor regarding the efficacy of this treatment is timely diagnosis and early initiation of therapy (5-6). Of note is that the damages caused by MPS is irreversible and treating after the establishment of these changes within the body tissue yields not benefit for sufferers. Almost all patients with MPS remain undiagnosed for a relatively long period of time without receiving appropriate treatment (7). To improve outcomes in patients with MPS, it is necessary to raise awareness among pediatricians, rheumatologists and pediatric rheumatologists about MPS disorders. Once It was reported that just a small proportion (under 20 percent) of these medical care providers and specialists could diagnose MPS accurately (8). So to raise suspicions about MPS and consequent investigations and interventions to avert irreversible damages, publishing various case reports on different clinical scenarios of MPS might be helpful. In the article, we describe two undiagnosed MPS patients with different clinical presentations.

\section{CASE REPORTS}

\section{CASE 1}

The first case is a 10-year old boy who developed wrist drop at the age of 9 years. He is the first child of relative parents and did not have any particular problem since birth and afterwards. Results of all the other clinical examinations were normal. He does not show cognitive impairment, goes to school and is intellectually normal. To find out the extent of nerves' involvement, electromyography (EMG) and nerve conduction velocity (NCV) tests were conducted. Right flexor carpi radialis, left abductor policis brevis and left opponens policis muscles had reduced or absent activity. Both median nerves had no sensory and motor activity. It was concluded that there was bilateral chronic severe median nerve injury above wrist. Brain MRI was normal. Biochemical routine laboratory tests were normal. There were not clinical symptoms for metal poisoning including lead and copper and ceruloplasmin, serum copper and lead level were within normal ranges. Then MPS was suspected. In urine testing, GAGs level was elevated. Electrocardiography and echocardiography was normal and ophthalmologic examination didn't show corneal clouding and any other abnormality.

Confirm the diagnosis, tandem mass spectrometry from dried blood spot was conducted. Five enzymes were checked. No Glycosaminoglycan alpha-L- iduronohydrolase activity was detected compatible with MPS I diagnosis. Genetic analysis revealed two homozygous missense mutations on p.Asp203Asn. After diagnosis, more evaluation for other abnormality were normal, including skeletal abnormality (by X-ray), abdominal ultrasonography and pulmonary function test.

\section{CASE 2}

The second case is an 8-year old boy with joint deformity and decreased range of motion predominantly in small joints of upper extremities. Decrease range of motion was detected in proximal interphalangeal (PIP) and distal interphalangeal (DIP) joints. Mild decrease in range of motion of elbow, knee and hip were also found. Pain was intermittent without particular pattern. There was no history of fever, rash and morning stiffness. This patient was primarily diagnosed as Juvenile idiopathic arthritis. He is the second child of relative parents delivered through normal vaginal delivery and his birthweight was $4 \mathrm{~kg}$. His first sibling is a healthy female. His symptoms were developed at 6 years of age. Patient's growth and development was normal. There was history of umbilical and inguinal hernias in 1 and 4 years old age, respectively. Routine biochemical tests were normal. No significant erosion was seen on X-ray. Abdominal ultrasonography revealed no abnormal findings. Echocardiography showed aortic insufficiency and mitral regurgitation. Ophthalmologic assessment yielded no defects. Absence of inflammatory signs and morning stiffness, involvement of large and small joints, involvement of small joints of upper extremities and relative parents raised the suspicion of metabolic disorders. Further urine evaluations showed raised GAGs level which was suggestive of MPS. Tandem mass spectrometry from dried blood spot was conducted. Decrease glycosaminoglycan Alpha-L-iduronohydrolase activity was detected. And genetic study confirmed the diagnosis of MPS I.

\section{DISCUSSION}

In this study we introduced two cases with musculoskeletal problems who were finally diagnosed with MPS I. This condition is a multi-systemic and progressive disorder which can lead to death, finally (9). This rare condition oc- 


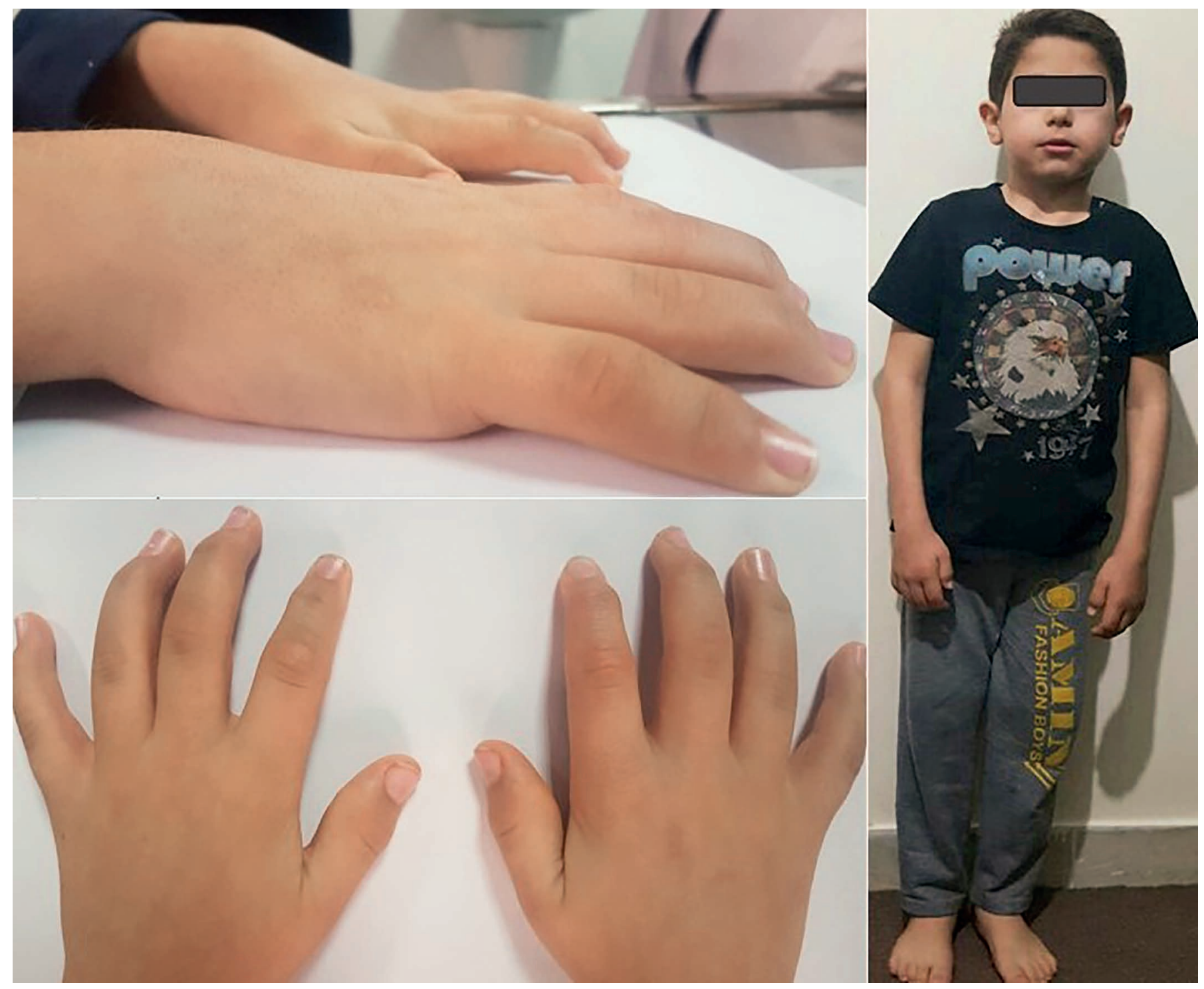

Fig. 1 Small joint contracture and normal facies in our patient with MPS type I.

curs in one out of 100,000 newborns. The inheritance pattern is via autosomal recessive pathway $(10,11)$; thus MPS is more prevalent in children of relative parents, as in our reported cases. The inherited genetic defect is responsible for deficiency of glycosaminoglycan alpha-L-iduronohydrolase. This defect impairs the degradation of GAGs and results in accumulation of GAGs in tissues which causes cellular damages. MPS type I is divided into three subgroups which include Scheie, Hurler-Scheie and Hurler syndromes. Scheie is the least severe and Hurler is the most severe form of the disease. Severe forms are usually diagnosed very early in the course of the disease due to obvious cognitive and mental impairment. They are not expected to live long and almost all of them die before 10 years of age without treatment. Hurler-Scheie patients are regarded as intermediate severity MPS. They may be free of mental impairment but still short life expectancy is seen due to cardio-pulmonary complications without treatment (2). The diagnostic problem emerges in the cases of Scheie syndrome who are the patients with the mildest form of MPS. These patients do not have characteristic facial features, cognitive impairment or any other specific symptom. Diagnosis in these patients is so diffi- cult due to the fact that clinical suspicion of MPS is not usually prompted (12). Although biochemical evaluations (urinary analysis) is so helpful but it is not sensitive in $100 \%$ of cases. To confirm the diagnosis, analysis of enzymatic activity is necessary and genetic assessment to figure out the responsible mutations is usually conducted (12).

The common symptoms of MPS can be summarized as joint contractures and skeletal deformities, cardiovascular involvement, corneal clouding, decreased visual acuity, hepatosplenomegaly, hernia and obstructive airway disease. As in our patients who presented with musculoskeletal manifestation, these complaints are the most common symptoms of MPS at the onset and course of the disease (13).

Considering neuropathy, wrist drop and foot drop are reported in Wilson's disease we assessed Wilson's disease and ruled out it $(14,15)$. All types of MPS except MPS types III and IX are associated with joint involvement (13). These complications are consequent to infiltration of soft tissues (ligaments, tendons, joint capsules, etc.) by GAGs. These changes lead to altered and malfunctioning skeletal alignment $(1,16)$. Musculoskeletal manifes- 
tations can mimic various rheumatologic disorders and lead to misdiagnosis such as juvenile idiopathic arthritis (13). The articular symptoms of MPS are not inflammatory-type. Stiffness is not maximum at morning and does not alter in severity with rest or activity. Tenderness, swelling and other signs of inflammation are not found. Inflammation markers are also not on the rise. Another point that proves non-inflammatory nature of MPS is that these symptoms do not improve with anti-inflammatory treatments $(13,17)$. Although any joint can be involved in severe types, articular symptoms mainly occur in phalangeal joints in MPS. Involvement of interphalangeal joints results in claw hand deformity and impairment of hand function $(7,12)$. While proximal interphalangeal (PIP) or metacarpophalangeal (MCP) joints involvement is more common in inflammatory conditions, DIP involvement is more probable in MPS $(13,18)$.

Carpal tunnel syndrome in children is very uncommon and should be carefully evaluated on presentation (19). Carpal tunnel syndrome is a common phenomenon in MPS patients which is responsible for more than $50 \%$ of carpal tunnel syndrome in pediatric patients. Infiltration of GAGs in flexor retinaculum and surrounding tissues of median nerves leads to thickening of these tissues and puts the nerve under pressure. Nerve compression is the underlying cause of carpal tunnel syndrome and related symptoms (19). Diagnosis of carpal tunnel syndrome faces two difficulties in MPS patients: The first one is that carpal tunnel syndrome might present with atypical manifestations in these patients. Pain and numbness may be absent as in our patients which was manifested with wrist drop. The second one is that probable cognitive impairment and mental retardation in MPS patients can delay the diagnosis $(20,21)$.

Another finding in one of our patients was histories of umbilical and inguinal hernia. Hernia raises clinical suspicion on several conditions such as hypothyroidism, trisomy 21 and MPS in pediatric patients. Hernias are among the most common extra-skeletal findings in MPS patients. For instance, A recent study has reported that $66 \%$ of MPS patients had umbilical and inguinal hernias (22). Most hernias need no surgical treatment except hernias with larger than 2 centimeters size. One of our patients had aortic insufficiency and mitral regurgitation on echocardiography. It is reported that cardiac symptoms, particularly mitral and aortic valve disease, murmur, cardiomyopathy, and cardiomegaly are common in MPS (23). Presence of extra-skeletal manifestation, especially those which are not common in pediatric age group, should be taken seriously as a clue for the diagnosis of metabolic disorders.

In this study, we used urine GAGs for screening MPS. Urine GAG tested by combination of semiquantitative Berry spot and 1,9-Dimethyl-Methylene Blue that have been used for the quantification of sulfated glycosaminoglycans. Berry spot test is not enough sensitive for MPS type III and IV (24), so we added 1,9-Dimethyl-Methylene Blue test in our center for screening and false negative has been reduced, seriously (25). It has low specific for diagnosis and after positive GAGs, enzymatic assay should be considered for confirmation.

\section{CONCLUSION}

Although musculoskeletal manifestation are common in a variety of clinical conditions, their presence at low ages or co-occurrence of other manifestations (such as cardiac, respiratory, CNS, etc) in multiple systems should prompt evaluation of patients for MPS and other metabolic disorders. Early diagnosis and treatment is necessary for prevention of irreversible damages in MPS patients. The rheumatologists' awareness on MPS should be promoted to achieve timely diagnosis and subsequent early treatment.

\section{ACKNOWLEDGMENTS}

We would like to thank parents' patients for giving permission to publish their data and pictures of these patients.

\section{CONFLICT OF INTEREST}

None

\section{REFERENCES}

1. Neufeld EU, Muenzer J. The mucopolysaccharidoses. In: Scriver CR (ed). The Metabolic and Molecular Bases of Inherited Disease. New York: McGraw-Hill, 2001; p. 3421-52.

2. Muenzer J. Overview of the mucopolysaccharidoses. Rheumatology (Oxford) 2011; 50(Suppl 5): v4-12.

3. Morishita K, Petty RE. Musculoskeletal manifestations of mucopolysaccharidoses. Rheumatology (Oxford) 2011; 50(Suppl 5): v19-25.

4. Petty RE, Southwood TR, Manners P, et al. International League of Associations for Rheumatology classification of juvenile idiopathic arthritis: second revision, Edmonton, 2001. J Rheumatol 2004; 31(2): 390-2.

5. Rosenthal J. Hematopoietic cell transplantation for storage diseases. In: Forman SJ, Negrin RS, Antin JH, Appelbaum FR (eds). Thomas' Hematopoietic Cell Transplantation: Stem Cell Transplantation. 5th Ed. Oxford: John Wiley \& Sons, 2016; p. 885-912.

6. Schulze-Frenking G, Jones SA, Roberts J, et al. Effects of enzyme replacement therapy on growth in patients with mucopolysaccharidosis type II. J Inherit Metab Dis 2011; 34(1): 203-8.

7. Vijay S, Wraith JE. Clinical presentation and follow-up of patients with the attenuated phenotype of mucopolysaccharidosis type I. Acta Paediatr 2005; 94(7): 872-7.

8. Manger B. Rheumatological manifestations are key in the early diagnosis of mucopolysaccharidosis type I. European Musculoskeletal Review 2008; p. 1-6.

9. Wraith JE. The mucopolysaccharidoses: aclinical review and guide to management. Arch Dis Child 1995; 72: 263-7.

10. Matte U, Yogalingam G, Brooks D, et al. Identification and characterization of 13 new mutations in mucopolysaccharidosistype I patients. Mol Genet Metab 2003; 78: 37-43.

11. Terlato N, Cox G. Can mucopolysacchari-dosis type I disease severity be predictedbased on a patient's genotype? A comprehen-sive review of the literature. Genet Med 2003; 5: 286-94.

12. Cimaz R, Vijay S, Haase C, et al. Attenuated type I mucopolysaccharidosis in the differential diagnosis of juvenile idiopathic arthritis: a series of 13 patients with Scheie syndrome. Clin Exp Rheumatol 2006; 24(2): 196-202.

13. Aldenhoven M, Sakkers RJ, Boelens J, et al. Musculoskeletal manifestations of lysosomal storage disorders. Ann Rheum Dis 2009; 68(11): 1659-65.

14. Rakesh N, Eison K, Shoval A, et al. The Mysterious Tale of Wilson's Disease Spasticity Presenting as a Radial Nerve Palsy: A case report. Am J Physical Med Rehab 2018; 97(3): a1-a131. (Abstract)

15. Aste R, Salaris E, Pische MG, et al. Mixed-type polyneuropathy in Wilson's Disease. Clin Neurophysiol 2013; 124(11): e204-e205. (Abstract)

16. Silveri CP, Kaplan FS, Fallon MD, et al. Hurler syndrome with special reference to histologic abnormalities of the growth plate. Clin Orthop Relat Res 1991; 269: 305-11. 
17. Cimaz R, Coppa GV, Kone-Paut I, et al. Joint contractures in the absence of inflammation may indicate mucopolysaccharidosis. Pediatr Rheumatol Online J 2009; 7: 18.

18. Bahadir C, Kurtulus D, Cihandide E. Mucopolysaccharidosis typeIS presenting with onset of carpal tunnel syndrome at adolescence. J Clin Rheumatol 2009; 15(8): 402-4.

19. al-Qattan MM, Thomson HG, Clarke HM. Carpal tunnel syndrome in children and adolescents with no history of trauma. J Hand Surg Br 1996; 21(1): 108-11.

20. Yuen A, Dowling G, Johnstone B, et al. Carpal tunnel syndrome in children with mucopolysaccaridoses. J Child Neurol 2007; 22(3): 260-3.

21. Van Heest AE, House J, Krivit W, Walker K. Surgical treatment of carpal tunnel syndrome and trigger digits in children with mucopolysaccharide storage disorders. J Hand Surg Am 1998; 23(2): 236-43.
22. Colmenares-Bonilla D, Colin-Gonzalez C, Gonzalez-Segoviano A, et al. Diagnosis of Mucopolysaccharidosis Based on History and Clinical Features: Evidence from the Bajio Region of Mexico. Cureus 2018; 10(11): e3617.

23. Braunlin EA, Stauffer NR, Peters CH, et al. Usefulness of bone marrow transplantation in the Hurler syndrome. Am J Cardiol 2003; 92(7): 882-6.

24. Chih-Kuang C, Shuan-Pei L, Shyue-Jye L, Tuen-Jen W. MPS screening methods, the berry spot and acid turbidity tests, cause a high incidence of false-negative results in Sanfilippo and Morquio Syndromes. J Clin Lab Analys 2002; 16: 253-8.

25. Abdi M, Hakhamaneshi MS, Alaei MR, et al. Validation of Urinary Glycosaminoglycans in Iranian patients with Mucopolysaccharidase type I: The effect of urine sedimentation characteristics. Iran J Child Neurol 2014; 8(4): 39-45. 